\title{
Il diritto futuro e la sua de-positivizzazione
}

\author{
The Future's Law and Its De-positivization
}

\author{
Mauro BARBERIS \\ Università di Trieste (Italia) \\ barberis@units.it
}

\begin{abstract}
Riassunto: L'unico modo per immaginare il futuro è pensarlo come prosecuzione del passato. II passato del diritto occidentale consiste in un processo di positivizzazione: dove 'positivo' significa contingente o mutevole, in modo che il diritto possa adattarsi sempre meglio a cambiamenti sempre più rapidi e imprevedibili. Peraltro, proprio nelle regioni più recenti del giuridico - diritto costituzionale, internazionale, comunitario - si sta verificando um processo contrario, che potrebbe chiamarsi de-positivizzazione. Nelle giurisprudenze di tutti questi settori, in altri termini, si vanno affermando principi spesso impliciti - uguaglianza, ragionevolezza, proporzionalità - che possono dirsi positivi solo in un censo debolissimo del termine: nel senso di costituire la parte comune, nella sua genericità, alla maggior parte delle dottrine normative della giustizia.
\end{abstract}

Parole chiave: diritto; futuro; de-positivizzazione; uguaglianza; ragionevolezza; proporzionalità; dottrine normative della giustizia.

\begin{abstract}
In order to imagine the law's future, we need reconstruct its past. The evolution of law in western countries disclaim a process of positivization: the law become and it is conceived as positive, i. e. less or more contingent available for change. In more recent areas of law, such as constitutional, international and European Union's law, However, inverses processes occur: the de-positivization of law. In constitutional case-law, in particular, an ever more crucial role is played by implicit norm as equality, reasonabless, proportionality. These principles are positive only in a very weak sense of the term: in reality, they are only a part, and a necessary part of the very concept of distributive justice.
\end{abstract}

Keywords: law; future; de-positivization; equality; reasonabless; proportionality; distributive justice.

Il passato è immaginario quanto il futuro Z. Bauman, L'ultima lezione

\section{PREMessa}

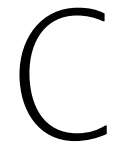

he futuro ha il diritto, ammesso ne abbia uno? La domanda resterebbe forse senza risposta se, come ci ha insegnato Reinhart Koselleck, il futuro non fosse relativo al passato ${ }^{1}$. Detto altrimenti, la narrazione del futuro non può che proseguire, in modo solo poco più congetturale, la narrazione del

1 Koselleck, R., Vergangene Zukunft (1979), trad. it.: Futuro passato: per una semantica dei tempi storici, Clueb, Bologna, 2007. 
passato. Qui di seguito, sulle tracce di Franco $V_{i o l a}^{2}$, provo a intrecciare le due narrazioni. I nodi sono gli stessi: costituzionalismo, pluralismo, giustizia. Ma il motivo che li tiene insieme è un altro: la de-positivizzazione del diritto.

Prima di iniziare, peraltro, bisogna forse precisare di quale diritto si racconta il passato e s'immagina il futuro. Ovviamente, non di qualunque insieme di norme che regga qualsiasi società, come nel motto ubi societas, ibi ius. Semmai, del diritto tipico, benché non esclusivo, della società occidentale, evolutosi attorno all'amministrazione della giustizia da parte di giudici ${ }^{3}$. Un fenomeno certo connesso a valori quali giustizia, rule of law e diritti ma, come questi, né universale ed eterno, bensì storicamente e culturalmente relativo.

\section{TRE CONCEZIONI DELLA POSITIVITÀ}

A ben vedere è sorprendente come il tema della positività, cruciale per intendere i tratti distintivi del diritto moderno e contemporaneo, sia trascurato dalla stessa teoria giuspositivista ${ }^{4}$. Proprio sulla positività del diritto, infatti, il discorso teorico giuspositivista si fa stranamente generico, e spesso lascia il posto al non-detto. Il diritto è positivo, si dice, nel senso di prodotto da uomini. Vero, ma chi altri potrebbe produrlo? È mai concepibile un diritto meramente naturale, ossia dato, necessario, magari immutabile?

Per mostrare come anche ciò che si chiama diritto naturale sia in definitiva un prodotto umano, storicamente e culturalmente relativo, il Gustav Radbruch giuspositivista e relativista usa questo esempio: l'acquisto della proprietà per occupazione della res nullius ${ }^{5}$. Che la cosa, mobile o immobile, spetti al primo occupante, e quindi la cacciagione al cacciatore e la pesca al pescatore, e così avanti, sembra dipendere dalla natura stessa delle cose: o forse, più perspicuamente, dalla definizione stessa dei termini impiegati.

2 Viola, F., «Il futuro del diritto», Persona y Derecho, 79 (2018, II) [en este mismo n ${ }^{\circ}$ ], pp. 9-36.

3 BARBeris, M., Europa del diritto. Sull'identità giuridica europea, Il Mulino, Bologna, 2008, e da ultimo ID., Una filosofia del diritto per lo stato costituzionale, Giappichelli, Torino, 2017.

4 Cfr. ZaCCaria, G. (a cura di), Diritto positivo e positività del diritto, Giappichelli, Torino, 1991, e VILLA, V., Conoscenza giuridica e concetto di diritto positivo, Giappichelli, Torino, 1993.

5 RADBRUCH, G., «La natura delle cose come forma giuridica di pensiero», Rivista internazionale di filosofia del diritto, 21, 1941, pp. 145-156; e BARBERIs, M., Manuale di filosofia del diritto, Giappichelli, Torino, 2011, pp. 67-69. 
In realtà, Radbruch mostra l'origine nient'affatto naturale, ma umana e specificamente consuetudinaria, di istituti siffatti: come David Hume, secoli prima, aveva spiegato l'origine della proprietà dalle aspettative reciproche dei soggetti. Certo, l'acquisto della res nullius per occupazione, in condizioni standard di scarsità limitata dei beni, pare istituto quasi necessario, senza alternative. Si pensi solo a regole diverse: la cosa appartiene all'intera comunità; non spetta ad alcuno; tocca al secondo occupante...

Ma si pensi, per contro, come l'istituto dell'acquisto della res nullius per occupazione perda la la propria apparenza di naturalità e/o di necessità appena cambiano le condizioni nelle quali è emerso. La disponibilità del bene può aumentare al punto da rendere vana l'appropriazione individuale; più spesso, può diminuire sino a renderne necessario il razionamento. Allora ci si accorge che la regola è solo apparentemente necessaria e/o naturale; in realtà, è contingente, muta secondo le situazioni e le esigenze dei soggetti.

Chi invocasse oggi una natura delle cose sempre uguale a se stessa, ad esempio reclamando un diritto naturale alla caccia o alla pesca, apparirebbe uno sperperatore delle risorse del pianeta ${ }^{6}$. Ma oltre alle situazioni, cambiano anche le esigenze dei soggetti. Una società più aperta e più dinamica di quella che credeva nel diritto naturale può affidarsi alla positività: la mutabilità o contingenza delle regole 7 . Positività che peraltro può essere ancora concepita in tre modi distinti, benché complementari.

In un primo senso, può dirsi positivo il solo diritto prodotto intenzionalmente («creato»), con l'intenzione di produrlo: paradigmaticamente, la legislazione. È questo il senso di 'positività' tipico della modernità: la contingenza radicale, la possibilità di creare il diritto letteralmente dal nulla. Si pensi alle mitologie rivoluzionarie del contratto sociale o del potere costituente: il sovrano creerebbe dal nulla un diritto puramente artificiale. Creazionismo comune nella tradizione giuspositivista: ma purtroppo, o per fortuna, implausibile ${ }^{8}$.

Persino il diritto codificato, da cui inizia lo stato legislativo, è irriducibile alla legislazione, ordinaria e speciale: l'unica fonte del diritto che somigli a questo primo modello di positività. Si pensi agli stessi codici, vero ideale dei riformatori illuministi e unica legge davvero generale. I codici non sono creati

6 Si veda Ostrom, E., Governing the Commons (1990), trad. it. Governare i beni collettivi, Marsilio, Venezia, 2006.

7 Luhmann, N., Ausdifferenzierung des Rechts (1981), trad. it. Il Mulino, Bologna, 1990.

8 BARBERIS, M., «Contro il creazionismo giuridico. Il precedente giudiziale fra storia e teoria», Quaderni fiorentini per la storia del pensiero giuridico moderno, 44 (2015), pp. 67-101. 
dal nulla, né sono il frutto della volontà sovrana, che si limita a disporne la redazione. Al contrario, essi sono l'esito di sistemazioni dottrinali e filosofiche della tradizione giurisprudenziale, e sono stabili, destinati a durare.

La prima concezione della positività esprime piuttosto la pretesa del legislatore moderno di stabilire lui le fonti del diritto. Si pensi all'art. 1 della Preleggi al Codice civile italiano del 1942, che elenca come fonti legislazione, regolamenti e consuetudini, ma che è stato presto superato dalla costituzione repubblicana del 1948 e dai trattati europei del 1956. Anche l'art. 1 Prel. escludeva dalle fonti la giurisprudenza e la dottrina: ossia un diritto positivo solo in un secondo senso di 'positività', come vediamo subito.

In un secondo senso, più debole, è positivo tutto il diritto prodotto da uomini: dunque, anche il diritto prodotto non intenzionalmente («evolutosi»), come conseguenza di atti umani intenzionalmente rivolti a scopi diversi che produrlo. Sono positive in questo senso la consuetudine, prodotta dai consociati per corrispondere ad aspettative di condotta reciproche, la dottrina (maggioritaria), prodotta dai giuristi al fine di insegnare il diritto, la giurisprudenza (costante), prodotta dai giudici al fine di risolvere controversie particolari.

Mentre il diritto positivo nel primo senso potrebbe essere prodotto anche da un legislatore singolo, con un solo atto, una volta nella storia del mondo, il diritto positivo nel secondo senso è il frutto degli effetti di composizione di molteplici atti individuali. Ci vogliono più giuristi per fare una dottrina (maggioritaria); occorrono più giudici per formare una giurisprudenza (costante). La fallacia creazionista o costruttivista consiste appunto nell'idea che diritto, lingua, mercato, possano ridursi alle intenzioni di qualcuno?

Che non tutto il diritto sia «formalmente stabilito» è stato riconosciuto da Alf Ross in relazione a consuetudini o precedenti ${ }^{10}$; lo stesso Herbert Hart, influenzato dalla tradizione di common law più di quanto si pensi, ha ammesso che almeno la norma di riconoscimento non può essere che una consuetudine. Ma la tradizione giuspositivista e giurealista diffida di queste idee: come se

9 HaYeK, F., Law, Legislation and Liberty (1973-79), trad. it. Il Saggiatore, Milano, 1986, specie pp. 200-202; sul carattere evolutivo delle istituzioni umane MENGER, K., Untersuchungen über die Methode der Sozialwissenschaften und der politischen Ökonomie insbesondere (1883), trad. it. Liberilibri, Macerata, 1996.

10 Ross, A., On Law and fustice (1952; 1958), trad. it. Einaudi, Torino, 1965, p. 96: «Una dottrina realistica delle fonti del diritto si fonda sull'esperienza ma riconosce che non tutto il diritto è positivo nel senso di 'formalmente stabilito'». 
'non intenzionale' significasse 'inconscio'; come se diritto, lingua, mercato, fossero prodotti in uno stato di sonnambulismo.

Eppure, un esempio di effetti non intenzionali dell'azione umana intenzionale si ha con la stessa interpretazione: l'attribuzione di significato a testi. I legislatori producono testi attribuendo loro certi significati; gli interpreti gliene attribuiscono altri, non necessariamente coincidenti con i primi. L'aspetto non intenzionale consiste appunto in questa possibile, e in realtà normale non-coincidenza: il diritto vivente, prodotto da dottrine maggioritarie e giurisprudenze costanti, costituisce uno sviluppo del diritto vigente.

Altro che inconscio o sonnambulismo, dunque: pensare all'interpretazione-prodotto come effetto non intenzionale dell'interpretazione-attività permette di spiegarla razionalmente applicandole, fra gli altri, gli strumenti della teoria dei giochi. Come ha mostrato Bruno Celano, autore che aveva già dissipato con strumenti analoghi le nebbie che gravano da sempre sulla consuetudine, l'interpretazione appare allora come un gioco nel senso della game theory: benché non cooperativo, bensì conflittuale ${ }^{11}$.

Nel secondo senso di 'positivo', al limite, potrebbe ritenersi positivo lo stesso diritto naturale, in quanto elaborato da giuristi, filosofi e teologi nella longue durée storica, uti universi, e non uti singuli. Per dare un senso alle idee stesse di positivizzazione e di depositivizzazione, peraltro, occorre forse postulare un terzo senso, debolissimo, di positività. Possono dirsi positive, cioè, anche esigenze etiche o pratiche generalissime, in ultima istanza storicamente e culturalmente relative ma rappresentative di intere epoche e culture.

La giustizia, l'eguaglianza, i diritti costituiscono, a un tempo, l'unico plausibile contenuto del diritto naturale e i maggiori candidati al ruolo di principi rappresentativi della cultura occidentale, almeno moderna: esigenze dell'etica o pratica tanto condivise da rappresentarne una sorta di grammatica profonda. Solo in società stabili e chiuse gli istituti giuridici possono apparire naturali o necessari; ma anche in società meno stabili e più aperte è possibile riconoscere forme di invarianza storica e culturale.

La positività del diritto, ricapitolando, è la sua contingenza più o meno radicale, secondo i sensi di 'positività' predicabili rispettivamente di legislazione, giurisprudenza e dottrina, e di giustizia, eguaglianza e diritti. Che il

11 Celano, B., Due problemi aperti della teoria dell'interpretazione giuridica, Mucchi, Modena, 2017. Sulle consuetudini cfr. invece ID., Fatti sociali, consuetudini, convenzioni, Aracne, Roma, 2010 . 
diritto abbia cominciato a cambiare e/o sia stato concepito come contingente, comunque, non è a sua volta una sorta di necessità storica ma solo l'esito di un processo, al contempo materiale e culturale, che può chiamarsi positivizzazione e che merita di essere considerato un po' più in dettaglio.

\section{PositivizZAZIONE}

'Diritto positivo' non è espressione usata dal legislatore. La stessa espressione 'positivismo giuridico', del resto, è solo un calco di 'positivismo filosofico', formatosi un secolo dopo la codificazione. Quando il legislatore italiano del 1942, all'art. 12, c. 2 Preleggi, ha voluto escludere che i principi generali del diritto potessero ricavarsi anche dal diritto naturale, non ha usato l'espressione 'diritto positivo'; ha mutuato invece da Santi Romano la locuzione, alquanto ridondante, 'ordinamento giuridico dello Stato'.

La positivizzazione del diritto - la concezione di questo come prodotto contingente di atti umani, ed eventualmente anche degli effetti non intenzionali di tali atti - è un processo storico, anch'esso prodotto da innumerevoli atti intenzionali e dagli ancora più innumerevoli effetti non intenzionali di tali atti. Già questo suggerisce che si tratti di un processo non solo materiale, ma anche culturale: ammesso che, per i fenomeni del mondo umano questa distinzione abbia senso. Ma può essere utile distinguerne tali due aspetti.

Come mero processo materiale - ossia astraendo dalla consapevolezza che possono averne gli attori - la positivizzazione è determinata dall'aumento della complessità sociale e dall'accelerazione del cambiamento prodottisi dall'antichità ai giorni nostri. Il diritto si è cioè trasformato progressivamente da fenomeno tradizionale o evolutivo, che stabilizzava in consuetudini le aspettative dei consociati esprimendosi in un discorso quasi-cognitivo, in legislazione, idonea a guidare il comportamento e formulata in termini normativi ${ }^{12}$.

Questo differenza è stata tematizzata dai filosofi greci ma non dai giuristi romani. Ius civile e ius naturale erano entrambi diritto umano, l'uno proprio della civitas, l'altro comune a differenti gentes: eppure, o proprio per questo, di positività non si parlava. La nozione fa la sua comparsa ai tempi di Tom-

12 In realtà, anche la legislazione continua a esprimersi in termini cognitivi: cfr. VILLEY, M., «De l'indicatif dans le droit», Archives de Philosophie du droit, 19 (1974), pp. 31-61 (anche in libro: Sirey, Paris, 1974). 
maso, con la gerarchizzazione del diritto in divino, naturale e positivo ${ }^{13}$ : dove il diritto positivo, mutevole a opera di autorità umane, gioca ancora un ruolo residuale.

La positivizzazione diventa processo anche culturale, cioè consapevole, con il giusnaturalismo volontarista e/o imperativista, che mira a privilegiare la legislazione sovrana rispetto a dottrina e giurisprudenza. La positività, intesa nel primo senso, assurge a marchio distintivo del diritto; ma dottrina e giurisprudenza, positive solo nel secondo senso, perdono carattere di fonti solo con la codificazione. Questa è sì parte di un progetto di modernizzazione della società, ma non è il culmine della positivizzazione del diritto.

Se, come ha mostrato Niklas Luhmann, la positivizzazione serve a rendere il diritto contingente, infinitamente modificabile, come richiesto da aumento della complessità e accelerazione del mutamento, allora il diritto naturale-razionale e la stessa codificazione diventano un impaccio. Il culmine della positivizzazione, paradossalmente, è la de-codificazione ${ }^{14}$ : il passaggio della produzione del diritto nelle mani del legislatore ordinario prima e dei governi poi, che la usano per la soluzione di qualsiasi problema sociale.

La de-codificazione genera iper-produzione e inflazione legislativa: ma non basta. Come osserva ancora Luhmann, nei processi di positivizzazione la richiesta di riforma si estende sino a dover essere soddisfatta anche dai giudici $^{15}$ : ma neppure questo basta. A intervenire con provvedimenti sempre più particolari e concreti devono essere pure agenzie amministrative, non solo nazionali e pubbliche. Così, lo spettro evocato dai liberali francesi dell'Ottocento, una società amministrata capillarmente dall'alto ${ }^{16}$, diventa la nostra realtà quotidiana.

La positivizzazione è anche un processo di istituzionalizzazione. Per assolvere all'immane compito regolativo richiestogli, cioè, al diritto servono sempre nuove istituzioni per produrlo, applicarlo, interpretarlo. In molti paesi, come la stessa Francia, tali istituzioni diventano una selva di sigle che si spartiscono fra loro l'intera esistenza umana, dalla culla alla bara. E questo, si badi, quando il diritto positivizzato funziona bene, come in Francia; quando funziona male, si è colti da una sorta di horror pleni.

13 Tommaso, Summa Theologiae II, I, qu. 91 e ss.

14 IRTI, N., L'età della decodificazione, Giuffrè, Milano, 1979.

15 Luhmann, N., Ausdifferenzierung des Rechts, trad. it. cit., p. 118.

16 Tocqueville, A. DE, La démocratie en Amérique (1840), Garnier-Flammarion, Paris, 1981, vol. II, p. 385. 
Evviva il diritto positivo, comunque. Ma il processo di positivizzazione ha un rovescio inquietante; lo Stato amministrativo del Novecento ha mostrato di potersi sempre trasformare in Stato totalitario. La sostituzione dello stato giurisdizionale pre-moderno con lo stato legislativo moderno è un progresso, ma presenta rischi strutturali, cui cerca di rimediare lo stato costituzionale post-moderno imponendo dei limiti alla positivizzazione ${ }^{17}$. Limiti abbastanza ampi, ormai, da prefigurare un processo inverso: la de-positivizzazione.

Il processo di positivizzazione del diritto, benché anch'esso storicamente e culturalmente relativo, è path-depending, ossia complessivamente irreversibile: a meno di eventi epocali, apocalittici e imprevedibili, beninteso. Eppure anche l'irreversibilità del processo conosce eccezioni e inversioni parziali, però vistose, in regioni del giuridico originariamente di confine ma oggi centrali e cruciali: diritto costituzionale, internazionale, dell'Unione europea... $\mathrm{E}$ soprattutto qui che s'incontrano processi di de-positivizzazione.

\section{De-PositivizZAZIONE}

La positività del diritto, s'è visto, equivale alla sua disponibilità per il legislatore: un rischio che, dopo Auschwitz, l'umanità non pare più disposta a permettersi. Nel mondo angloamericano, anche dove, come nel Regno Unito, resiste il principio tipico dello stato legislativo, la sovranità del Parlamento, il diritto positivo intenzionale è temperato da uno non intenzionale: il common law (cfr. qui $\$ 2$ ). Il constitutionalism, la garanzia dei sistemi politici e giuridici anglosassoni contro le derive totalitarie, consiste anche in questo.

I sistemi giuridici continentali hanno peraltro superato questo stadio evolutivo codificando il diritto e adottando una dottrina delle fonti che esclude il diritto giurisprudenziale. Qui, le uniche garanzie contro il totalitarismo, sino alla fine della seconda guerra mondiale, sono state interne allo stato legislativo: principio di legalità, riserva di legge... Garanzie illusorie, sinché la legge può assumere qualsiasi contenuto: come peraltro deve necessariamente fare, se vuole corrispondere a domande di regolazione sempre crescenti.

17 Questa terminologia - specie l'uso di 'stato', con la minuscola, a indicare una situazione, uno stadio dell'evoluzione del diritto - è proposta sistematicamente in BARBERIS, M., Una filosofia del diritto per lo stato costituzionale, cit. Ma cfr. già FIORAVANTI, M., «Stato e costituzione», in ID. (a cura di), Lo Stato moderno in Europa, Laterza, Roma-Bari, 2002, pp. 8-21, e FERRAjOLI, L., La democrazia attraverso $i$ diritti, Laterza, Roma-Bari, 2013, pp. 5-11. 
Garanzie ulteriori sono fornite dallo stato costituzionale: costituzioni rigide e lunghe, controllo di costituzionalità, irradiazione dei principi costituzionali in tutto il corpo del diritto tramite la cosiddetta interpretazione costituzionale. Come vediamo subito, ognuno di questi istituti, uti singoli e in linea di principio, è funzionale alla de-positivizzazione del diritto: l'attività legislativa si trasforma da arbitraria in meramente discrezionale. Uti universi e di fatto, però, tali istituti producono sistemi sempre più complessi e intrinsecamente fragili: come vediamo subito dopo.

La rigidità costituzionale, e la resistenza al cambiamento di accordi e trattati internazionali in tema di diritti, producono i tipi di de-positivizzazione tematizzati da Ernesto Garzón Valdéz e da Luigi Ferrajoli in termini, rispettivamente, di coto vedado ${ }^{18}$ e di sfera dell'indecidibile: la seconda nella duplice forma di sfere dell'indecidibile che (la legge non può violare i diritti di libertà), e indecidibile che non (la legge non può non attuare i diritti sociali) ${ }^{19}$. Entrambe le sfere sottraggono al legislatore interi àmbiti di decisione.

Il controllo di costituzionalità, invece, reagisce con la disapplicazione o con la nullità alla violazione dei diritti costituzionalmente stabiliti: ma lo fa in forme giudiziali, dunque su impulso di altre istituzioni, con decisioni motivate, benché definitive, e soprattutto previa interpretazione, sia della legislazione sia della costituzione. I vari tribunali costituzionali e internazionali, infatti, si sono sviluppati meno nella forma originaria di legislatori negativi che in quella, auto-attribuitasi, di interpreti specializzati ${ }^{20}$.

Come vedremo meglio all'ultima sezione, infatti, l'esercizio di funzioni di legislazione negativa è solo il rimedio estremo attribuito dai costituenti al giudice delle leggi, in alternativa alla conservazione della legge. Ma nell'adempimento di tale funzione, i tribunali costituzionali e internazionali hanno finito per attribuirsene un'altra, che oggi può considerarsi prevalente e talvolta, specie in campo internazionale, addirittura l'unica: l'interpretazione della costituzione e dei trattati, soprattutto in materia di diritti.

L'irradiazione dei principi costituzionali e internazionali nell'intero corpo del diritto, interno e/o internazionale, tramite l'interpretazione costituzionale, è la terza condizione per l'affermarsi dello stato costituzionale odierno: condizione disgiuntamente necessaria, e sufficiente solo congiuntamente

18 GarZón VALdÉs, E., «Democracia y representación», Doxa, 6 (1989), pp. 143-163.

19 Ferrajoli, L., La democrazia attraverso $i$ diritti, cit., pp. 48-49.

20 Si vedano i lavori raccolti in KELSEN, H., La giustizia costituzionale, Giuffrè, Milano, 1981. 
con le altre. L'attribuzione o auto-attribuzione ${ }^{21}$ ai tribunali costituzionali o internazionali dell'autorità di determinare in ultima istanza il significato delle leggi produce cambiamenti anche nelle funzioni di altri organi istituzionali.

Legislatore, o governi che esercitano l'iniziativa legislativa, cominciano a produrre leggi idonee a superare il controllo di costituzionalità; si inizia a parlare, a questo proposito, di discrezionalità legislativa, quasi che il legislatore dovesse giustificarsi di fronte al costituente come l'amministratore dinanzi al legislatore. La legge, rigorosamente non motivata nello stato legislativo, viene dotata di preamboli che ne enunciano i principi ispiratori e che divengono, così, una sorta di motivazione.

Lo stato costituzionale non è solo enormemente più complesso dello stato legislativo, è anche intrinsecamente fragile. Basti dire che i suoi organi esercitano funzioni diverse, talora opposte, a quelle attribuite loro nello stato legislativo. L'indirizzo politico e spesso anche l'iniziativa delle leggi è da tempo passata al (cosiddetto) esecutivo; il (cosiddetto) legislativo esercita ormai soprattutto funzioni di controllo sul governo; il (cosiddetto) giudiziario produce ormai pacificamente diritto per via d'interpretazione.

Come nei sistemi di cheks and balances, retti da una sorta di mano invisibile istituzionale, anche il sistema dello stato costituzionale diviene opaco, né a renderlo più trasparente vale la sua integrazione nell'Unione europea. A entrambi i livelli, le organizzazioni sociali che assicuravano il funzionamento dello stato legislativo, come partiti, sindacati, stampa indipendente, perdono peso a favore di apparati politicamente irresponsabili: alte burocrazie, gruppi finanziari, lobbies, gestori del web, servizi di sicurezza...

Settori sempre più ampi della società sono penalizzati dalle ricorrenti crisi economiche e ancor più dai processi di modernizzazione e razionalizzazione, che producono concentrazioni di ricchezza e diseguaglianze ormai comparabili solo a quelle dell'ancien régime. I settori penalizzati percepiscono la stessa penalizzazione, tramite i nuovi media, come complotti perpetrati dall'establishment ai loro danni, e si affidano a leader e movimenti capaci di interpretare il disagio, ma anche di piegarlo ai propri interessi ${ }^{22}$.

21 Troper, M., «Marshall, Kelsen, Barak and the Constitutionalist Fallacy», International fournal of Constitutional Law, 3/1 (2005), pp. 24-38.

22 E la dinamica del cosiddetto populismo: cfr., da ultimo MudDE, C., On Extremism and Democracy in Europe and the Populist Radical Right. A Reader, Routledge, Abingdon-on-Thames (UK), 2017, e la parte monografica di «Teoria politica», 7, 2017, intitolata «Populismi», pp. 23-182. 
Specie nei paesi di democrazia più recente, del resto, lo stato costituzionale è sempre stato guardato con sospetto; i tribunali costituzionali spesso sono percepiti come una parte dell'establishment e un sostegno per l'esecutivo $^{23}$. Anche altrove, d'altronde, la sfera dell'indecidibile è soggetta a erosione. I diritti di libertà sono esposti ai processi di securizzazione innescati da terrorismo e immigrazione ${ }^{24}$; i diritti sociali sono strutturalmente soggetti alla non attuazione, per mancanza di rimedi all'inazione dei governi.

Forse lo stato costituzionale si sarebbe già trasformato nel potere immenso e tutelare intravisto secoli fa da Tocqueville se non fosse per il suo triplice pluralismo. Pluralismo dei valori, anzitutto: le costituzioni esprimono i valori confliggenti di società multiculturali. Pluralismo istituzionale, poi: poteri pubblici, privati e del terzo settore resistono a ogni tentativo di monopolizzazione. Pluralismo giuridico, infine: il diritto è prodotto, applicato e interpretato da una pluralità incontrollabile di istituzioni giuridiche.

\section{De-POSITIVIZZAZIONE COSTITUZIONALE}

L'espressione più vistosa della de-positivizzazione è l'interpretazione costituzionale: locuzione che copre tutt'e tre le fasi del processo. Prima, interpretazione in senso stretto: attribuzione di significati alla costituzione e di significati conformi alle leggi. Poi, interpretazione nel senso lato d'integrazione: produzione di norme costituzionali implicite. Infine, interpretazione nel senso latissimo di argomentazione: fra tali norme acquistano un ruolo cruciale principi o argomenti come eguaglianza, ragionevolezza, proporzionalità.

Mentre l'interpretazione non fa che attribuire significato a disposizioni positive nel senso forte di contingentemente stabilite, l'integrazione produce norme implicite, positive solo nel senso debole di prodotte dagli stessi giudici. Ma, fra queste, argomenti o principi come eguaglianza, ragionevolezza e proporzionalità paiono positivi solo nel senso debolissimo di corrispondenti a invarianti culturali. Si tratta infatti di altrettante specificazioni del concetto di giustizia distributiva, comune a tutte le sue concezioni ${ }^{25}$.

23 Nino, C. S., «A Philosophical Reconstruction of Judicial Review», Cardozo Law Review, 14 (1992-1993), pp. 799-846.

24 Cfr. Buzan, B.; Waever, O.; De Wilde, J., Security. A New Framework for Analysis, Lynne Rienner, London, 1998.

25 Rawls, J., A Theory of fustice (1971), trad. it. Feltrinelli, Milano, 1982, p. 23. 
Questo processo s'è svolto in forme diverse in contesti differenti, interni e internazionali, ma ha prodotto principi abbastanza simili da essere formulati, ad esempio, in documenti come la Carta di Nizza ${ }^{26}$, e da far parlare di dialogo fra le corti, comparativismo costituzionale, migrazione d'istituti, e simili. Qui fornisco solo un modello del processo: uno schema evolutivo troppo semplice, si badi, per corrispondere fedelmente anche solo all'evoluzione della giurisprudenza costituzionale italiana, alla quale pure si ispira ${ }^{27}$.

Il primo passaggio del processo di de-positivizzazione costituzionale è l'interpretazione conforme alla costituzione di disposizioni legislative sospette di incostituzionalità. È appena il caso di notare come già l'interpretazione che «adegua» la legge alla costituzione rappresenti un esito non intenzionale, nel senso sopra detto, del meccanismo progettato dai costituenti. Questi, infatti, avevano previsto solo due possibilità: la Corte costituzionale avrebbe dovuto limitarsi ad annullare la norma di legge oppure lasciarla in vigore.

Messi di fronte a quest'alternativa secca, peraltro, i giudici costituzionali, non solo italiani, hanno spesso scelto una terza possibilità. Essi hanno cioè interpretato le disposizioni rispettivamente costituzionale e legislativa in modo da adeguare la seconda alla prima, così salvandola da una dichiarazione d'incostituzionalità, ma lasciandola in vigore nonostante la sua ambiguità. Dagli anni Ottanta in poi, anzi, la Corte costituzionale italiana ha consumato una rottura ancora più netta con il sistema progettato dal costituente.

I giudici costituzionali hanno cioè imposto ai giudici ordinari, a pena d'inammissibilità della questione da essi sollevata, di cercare loro l'interpretazione conforme, rimettendo la questione alla Corte solo ove non ne trovino alcuna. In questo modo, non solo la Corte ha decentrato ai giudici ordinari un controllo di costituzionalità originariamente accentrato, ma li ha anche implicitamente invitati a interpretare tutta la legislazione in conformità ai principi costituzionali: irradiando così questi ultimi nell'intero corpo del diritto ${ }^{28}$.

Il secondo passaggio del processo di de-positivizzazione costituzionale aggiunge all'interpretazione conforme di legge e costituzione l'integrazione del-

26 Si veda l'intero capo III, intitolato «Uguaglianza», e l'enunciazione del principio di proporzionalità nell'art. 52, fra le «disposizioni generali» finali.

27 Il modello costituisce un'universalizzazione ma anche una correzione, di quello proposto in BARBERIs, M., «Eguaglianza, ragionevolezza e diritti», Rivista di filosofia del diritto, 2 (2013/1), pp. 191-204.

28 Processo chiamato irradiazione da Robert Alexy, e costituzionalizzazione da Riccardo Guastini, in lavori troppo noti per doverli citare ulteriormente. 
la stessa costituzione, affollandola di norme implicite. La distinzione fra norme rispettivamente esplicite e implicite è discussa e discutibile: specie ove la si consideri una distinzione assoluta, come avviene di solito, e non meramente relativa ${ }^{29}$. Si tende a pensare, cioè, che vi siano norme esplicite, prodotte solo dal legislatore, e norme implicite, prodotte solo dai giudici ${ }^{30}$.

Tuttavia, ispirandosi a movimenti post-realisti nordamericani come il Legal process, o anche, più vicino a noi, a Hans Kelsen, è possibile relativizzare questa distinzione: tutte le norme, anche le norme implicite, sono di produzione tanto legislativa quanto giudiziale. Sincronicamente, astraendo dal tempo, una stessa disposizione può avere significati espliciti, in maggior misura legislativi, e impliciti, in maggior misura giudiziale: ma solo in maggior misura, perché le disposizioni sono prodotte comunque dal legislatore.

Diacronicamente, nel tempo, una norma che nel momento $\mathbf{x}$ può apparire implicita, nel momento $\mathbf{y}$ può diventare esplicita, ove una giurisprudenza costante la attribuisca pacificamente alla disposizione come suo significato. Qui, dunque, intenderò per norme implicite significati attribuiti a una o più disposizioni già dotate di altri significati: sensi che sono norme esplicite perché da sempre o almeno costantemente sono attribuiti come significato alla disposizione. Nel sistema costituzionale queste norme implicite hanno due usi principali.

In entrambi i casi si tratta di principi, cioè di norme generiche, ricavati da una o più disposizioni e, se ricavati da una sola, ulteriori alle norme già espresse pacificamente da questa. Il primo uso dei principi li impiega per tutelare diritti impliciti o non enumerati, quali quelli menzionati dall'art. 2 Cost. it., o dal nono emendamento della costituzione federale statunitense ${ }^{31}$. In Italia, ad esempio, sono diritti o principi impliciti, privi di una loro specifica disposizione, dignità umana, rispetto della vita, laicità dello Stato, sicurezza...

Anche tali diritti, nella loro genericità, si prestano a fungere da criteri per l'attribuzione di (altri) diritti: attribuzione che non può pregiudicare, appunto,

29 Per una discussione fra Guastini, Giorgio Pino ed Enrico Diciotti sulle norme implicite, da cui qui si cerca di trarre alcune conclusioni, si veda VelLuZZI, V. (a cura di), «Interpretazione e costruzione del diritto. Riflessioni su Interpretare e argomentare di Riccardo Guastini», Rivista di filosofia del diritto, 2 (2013/1), pp. 73-136.

30 GuastinI, R. «Replica», Rivista di filosofia del diritto (2013/1), p. 136: «Mi sembra di grande interesse $[. .$.$] sapere se una data norma sia, diciamo così, di fonte legislativa o invece di fonte$ giurisprudenziale».

31 «La Repubblica riconosce e garantisce i diritti inviolabili dell'uomo» (art. 2 Cost. it.); «Il fatto che la Costituzione enumeri determinati diritti non potrà intendersi nel senso di negare o svilire altri diritti che il popolo si sia riservato» (nono emendamento alla Costituzione Federale Statunitense). 
dignità, vita, laicità, sicurezza... Il secondo uso, per converso, riguarda principi impliciti che possono anch'essi tutelare meri diritti, ma che sono tipicamente impiegati come meta-diritti: criteri per l'attribuzione di altri diritti ${ }^{32}$. Fungono paradigmaticamente da meta-diritti, in Italia, i significati attribuiti all'art. 3, c. 1, Cost., a partire dall'unico significato espresso letteralmente dalla disposizione ${ }^{33}$.

Letteralmente, l'art. 3, c. 1 Cost. it. esprime solo un divieto di discriminare fra soggetti per sette ragioni specificamente enumerate (sesso, razza, religione...). Divieti di discriminazione siffatti si trovano in tutte le costituzioni e talora anche in trattati internazionali. Mentre però i giudici internazionali, o anche costituzionali che giudicano direttamente casi concreti hanno meno occasioni di usarli, essi trovano largo impiego da parte di giudici della costituzionalità di leggi per assicurare l'eguale distribuzione di diritti da parte del legislatore.

I giudici delle leggi non possono certo sostituirsi al legislatore, ma possono controllare l'esercizio eguale della sua discrezionalità, e dunque la coerenza interna di essa. A questo scopo, l'enumerazione delle ragioni di cui all'art. 3, c. 1 Cost. it. è stata ritenuta non tassativa, estendendo il divieto a qualsiasi discriminazione. S'è così prodotta la norma, originariamente implicita, che vieta di discriminare soggetti eguali, ma anche di assimilarne di diversi, e anzi di discriminare o assimilare (non solo soggetti, ma) casi, diversi o simili.

È questo il principio di eguaglianza: il nucleo del concetto stesso di giustizia. Secondo la dottrina standard della giustizia distributiva, infatti, questa consiste di due parti, una fissa, necessaria, e una mobile, contingente. La prima, fissa, è rappresentata appunto dal principio di eguaglianza: il trattamento eguale di casi eguali, e diverso di casi diversi. Una seconda parte, mobile, è rappresentata dalle formule di giustizia, quali «a ognuno secondo i meriti», o i bisogni, o la sorte, che specificano cosa intendere per 'uguale' e 'diverso' ${ }^{34}$.

La de-positivizzazione, qui, nasce come effetto non intenzionale del controllo di legittimità. La Corte non può sostituirsi al legislatore, scegliendo le formule di giustizia: la parte contingente del concetto. La Corte può solo

32 Guastini, R., La sintassi del diritto, Giappichelli, Torino, 2011, pp. 97-98, e ID., Distinguendo ancora, Marcial Pons, Madrid-Barcelona, 2013, pp. 255-266.

33 «Tutti i cittadini hanno pari dignità sociale e sono eguali davanti alla legge, senza distinzione di sesso, di razza, di lingua, di religione, di opinioni politiche, di condizioni personali e sociali» (art. 3. C. 1 Cost. it.).

34 Cf. ad esempio Ross, A., Law and fustice (1958), trad. it. Einaudi, Torino, 1965, pp. 254-260. 
controllare l'eguale trattamento dei casi: la parte necessaria. Per un altro effetto non previsto dal costituente, il parametro più invocato nel controllo di costituzionalità è divenuto il principio di eguaglianza: norma implicita, e anzi positiva solo nel senso debolissimo visto a suo tempo ( $\$ 2$ in fine).

In base a tale principio, il legislatore sceglierebbe come distribuire i diritti, ma così facendo si vincolerebbe a trattare ugualmente casi uguali, e diversamente casi diversi da quello regolato. L'eguaglianza diviene così un requisito interno a qualsiasi distribuzione di diritti da parte del legislatore: requisito che dipende più dalla struttura stessa del concetto di giustizia che dalla volontà del costituente. È come se la Corte avesse «appeso» il principio di eguaglianza al «gancio» della disposizione che meglio vi si prestava, l'art. 3, c. 1 .

$\mathrm{Si}$ noti come questa interpretazione, originariamente un'integrazione della disposizione, si aggiunga alla prima: l'art. 3, c.1, cioè, esprime un divieto di alcune discriminazioni puntuali più un divieto di tutte le altre discriminazioni, il principio di eguaglianza. Ma poiché distinguere caso da caso è proprio il compito del legislatore, ecco che la disposizione riceve un'ulteriore integrazione, oggi interpretazione: il legislatore può operare tutte le distinzioni che vuole salvo quelle irragionevoli (principio di ragionevolezza) $)^{35}$.

Sin qui, la Corte aveva solo controllato se la discrezionalità del legislatore, esercitata in un caso, fosse coerente con il suo esercizio in un altro caso: il cosiddetto tertium comparationis, terzo oltre al parametro e all'oggetto del controllo $^{36}$. Questo limite era già più apparente che reale: è sempre possibile, infatti, invocare un caso rispetto al quale il legislatore appaia incoerente. Ma anche questo limite apparente cade ove il legislatore, invece di distinguere, assimili irragionevolmente casi: qui non c'è più alcun tertium, neppure fittizio ${ }^{37}$.

Con l'adozione del parametro della ragionevolezza, ancor più disperatamente generico dell'eguaglianza, la Corte inizia a esercitare un controllo di costituzionalità non più triadico (parametro, oggetto e tertium) ma meramente diadico: a essere controllata è cioè la (ir)ragionevolezza intrinseca della legge. Qui la Corte sembra passare da un controllo di legittimità relativo alla coerenza interna della discrezionalità del legislatore, a un controllo di merito, esterno e assoluto, sulla (ir)ragionevolezza della legge.

35 Sorrentino, F., Eguaglianza. Lezioni raccolte da E. Rinaldi, Giappichelli, Torino, 2011, p. 12. Sul carattere proteiforme, irriducibile della ragionevolezza ModugnO, F., La ragionevolezza nella giustizia costituzionale, Scientifica, Roma, 2007.

36 Paladin, L., Il principio costituzionale di uguaglianza, Giuffrè, Milano, 1965.

37 SORRENTino, F., Eguaglianza, cit., p. 40. 
Questo terzo passaggio della de-positivizzazione costituzionale, già largamente compiuto nella giurisprudenza costituzionale e internazionale tedesca ed europea, ma solo in corso in quella italiana, può farsi coincidere con l'adozione del principio di proporzionalità, come specificazione e articolazione ulteriore della ragionevolezza. La (ir)ragionevolezza della legge viene cioè controllata direttamente, diadicamente, specificandola in tre o quattro test fra loro successivi: legittimità, idoneità, necessità, proporzionalità in senso stretto $^{38}$.

Il principio di proporzionalità prevede che diritti individuali possano sacrificarsi a interessi collettivi solo rispettando quattro parametri: di cui peraltro il primo, la legittimità costituzionale degli interessi perseguiti, si dà spesso per scontato, per una ragione che ha anch'essa a che fare con la de-positivizzazione. Le costituzioni dello stato costituzionale, infatti, sono tendenzialmente complete: vi si trova sempre un principio, se non esplicito almeno implicito, che giustifichi il perseguimento di qualsiasi interesse.

Il primo test di proporzionalità è così il controllo di adeguatezza, analogo al controllo di razionalità di cui si parla anche in sede di controllo di ragionevolezza. Per superare il controllo di adeguatezza, i mezzi scelti dal legislatore devono cioè essere idonei a conseguire il fine perseguito: benché, in materie tecniche o riservate ai governi, spesso solo lo stesso governo possa valutarne l'idoneità. Eppure, misure che sacrifichino diritti a fini vaghi, simbolici o pretestuosi non sono affatto casi di scuola, specie in materia di sicurezza.

Il secondo test di proporzionalità è il controllo di necessità, al quale di regola si procede solo ove il primo abbia dato esito favorevole: di regola, perché in materia penale può essere opportuno valutare prima la necessità del sacrificio della libertà e poi l'adeguatezza ${ }^{39}$. Il sacrificio dei diritti individuali, secondo tale test, dev'essere il minimo indispensabile al conseguimento del fine. $\mathrm{Si}$ tratta di test più stringente del precedente, ma che comporta presa in considerazione, da parte della Corte, di alternative meno onerose per i diritti.

38 Cohen-Eliya, M.; Porat, I., Proportionality and Constitutional Culture, Cambridge UP, Cambridge (Mass.), 2013, e art. 52 della Carta dei diritti fondamentali dell'Unione europea (Bruxelles/ Nizza 2000): «Nel rispetto del principio di proporzionalità, possono essere apportate limitazioni [all'esercizio dei diritti e delle libertà] solo ove siano necessarie e rispondano effettivamente a finalità di interesse generale riconosciute dall'Unione [...]».

39 Cf. Troncon, P., Riflessioni sull'uso del canone di proporzionalità nell'azione legislativa contemporanea in materia penale, inedito. 
Il terzo test di proporzionalità in senso ampio è il controllo di proporzionalità in senso stretto: ammesso che il sacrificio di diritti sia adeguato e necessario al conseguimento del fine costituzionalmente legittimo, potrebbe ancora darsi sproporzione fra mezzi e fini. È a proposito di quest'ultimo test che si parla comunemente di bilanciamento o ponderazione: metafora che esplicita ulteriormente il carattere consequenzialistico dei tre test, risalenti alla lunga stagione della prevalenza dell'utilitarismo nell'etica occidentale.

Il bilanciamento si presta a due obiezioni, una radicale e una moderata. L'obiezione più radicale, tipica del pluralismo dei valori, è che i valori sono incommensurabili, privi di misura comune. L'obiezione moderata, tipica del liberalismo politico, è che valori deontologici come i diritti individuali vadano sottratti al bilanciamento con interessi collettivi. In realtà, nella giurisprudenza delle corti costituzionali e internazionali, emerge questa soluzione: diritti e interessi si bilanciano, ma con una presunzione vincibile a favore dei primi ${ }^{40}$.

\section{Conclusione}

La conclusione che può trarsi da tutte queste vicende è duplice. Se i processi di de-positivizzazione in atto proseguiranno, è probabile che la tensione interna allo stato costituzionale, fra poteri politici legittimati democraticamente e poteri giudiziali legittimati solo dal diritto e/o dai diritti, produca conflitti, ed è possibile che questi portino a crisi dello stato costituzionale. In particolare, è possibile un ridimensionamento del controllo giudiziale, anche internazionale, come quello cui si assiste in paesi come la Turchia.

La de-positivizzazione potrebbe dunque fermarsi, specie se si affermeranno i populismi, sovranismi e isolazionismi che oggi si affacciano. Ma anche con questa cautela, è possibile prevedere che all'aumento della complessità continuerà a corrispondere un aumento della richiesta di diritto alle Corti, e dunque una ulteriore de-positivizzazione. Anzi: l'esito paradossale dell'affermarsi di élites politiche sempre più incolte giuridicamente potrebbe attribuire al giudiziario il ruolo di ultimo contrappeso del potere.

40 BARBERIS, M., Non c'è sicurezza senza libertà. Il fallimento delle politiche antiterrorismo, Il Mulino, Bologna, 2017. 


\section{REFERENCIAS}

BArberis, M., Europa del diritto. Sull'identità giuridica europea, Il Mulino, Bologna, 2008.

- Manuale di filosofia del diritto, Giappichelli, Torino, 2011.

— «Eguaglianza, ragionevolezza e diritti», Rivista di filosofia del diritto, 2 (2013/1), pp. 191-204.

— «Contro il creazionismo giuridico. Il precedente giudiziale fra storia e teoria», Quaderni fiorentini per la storia del pensiero giuridico moderno, 44 (2015).

- Non c'è sicurezza senza libertà. Il fallimento delle politiche antiterrorismo, Il Mulino, Bologna, 2017.

- Una filosofia del diritto per lo stato costituzionale, Giappichelli, Torino, 2017.

Buzan, B.; Waever, O.; De Wilde, J., Security. A New Framework for Analysis, Lynne Rienner, London, 1998.

Celano, B., Fatti sociali, consuetudini, convenzioni, Aracne, Roma, 2010.

- Due problemi aperti della teoria dell'interpretazione giuridica, Mucchi, Modena, 2017.

Cohen-Elrya, M.; Porat, I., Proportionality and Constitutional Culture, Cambridge UP, Cambridge (Mass.), 2013.

FERrajoli, L., La democrazia attraverso i diritti, Laterza, Roma-Bari, 2013.

FioraVANTI, M., «Stato e costituzione», in Fioravanti, M. (a cura di), Lo Stato moderno in Europa, Laterza, Roma-Bari, 2002.

GARZÓN VALDÉs, E., «Democracia y representación», Doxa, 6 (1989), pp. 143-163.

GUASTINI, R., La sintassi del diritto, Giappichelli, Torino, 2011.

- Distinguendo ancora, Marcial Pons, Madrid-Barcelona, 2013.

— «Replica», Rivista di filosofia del diritto (2013/1).

HAYEK, F., Law, Legislation and Liberty (1973-79), trad. it. Il Saggiatore, Milano, 1986.

IRTI, N., L'età della decodificazione, Giuffrè, Milano, 1979.

Kelsen, H., La giustizia costituzionale, Giuffrè, Milano, 1981.

Koselleck, R., Vergangene Zukunft (1979), trad. it. Futuro passato: per una semantica dei tempi storici, Clueb, Bologna, 2007.

Luhmann, N., Ausdifferenzierung des Rechts (1981), trad. it. Il Mulino, Bologna, 1990.

MENGER, K., Untersuchungen über die Methode der Sozialwissenschaften und der politischen Ökonomie insbesondere (1883), trad. it. Liberilibri, Macerata, 1996.

Modugno, F., La ragionevolezza nella giustizia costituzionale, Scientifica, Roma, 2007.

Mudde, C., On Extremism and Democracy in Europe and the Populist Radical Right. A Reader, Routledge, Abingdon-on-Thames (UK), 2017.

NinO, C. S., «A Philosophical Reconstruction of Judicial Review», Cardozo Law Review, 14 (1992-1993), pp. 799-846.

Ostrom, E., Governing the Commons (1990), trad. it. Governare i beni collettivi, Marsilio, Venezia, 2006.

PALADIN, L., Il principio costituzionale di uguaglianza, Giuffrè, Milano, 1965.

RADBRUCH, G., «La natura delle cose come forma giuridica di pensiero», Rivista internazionale di filosofia del diritto, 21, 1941.

RaWLs, J., A Theory of fustice (1971), trad. it. Feltrinelli, Milano, 1982.

Ross, A., Law and Fustice (1958), trad. it. Einaudi, Torino, 1965. 
Ross, A., On Law and fustice (1952; 1958), trad. it. Einaudi, Torino, 1965.

Sorrentino, F., Eguaglianza. Lezioni raccolte da E. Rinaldi, Giappichelli, Torino, 2011.

Tocqueville, A. DE, La démocratie en Amérique (1840), Garnier-Flammarion, Paris, 1981, vol. II.

Tommaso, Summa Theologiae II, I, qu. 91 e ss.

TronCON, P., Riflessioni sull'uso del canone di proporzionalità nell'azione legislativa contemporanea in materia penale, inedito.

Troper, M., «Marshall, Kelsen, Barak and the Constitutionalist Fallacy», International Fournal of Constitutional Law, 3/1 (2005), pp. 24-38.

VELluZZI, V. (a cura di), «Interpretazione e costruzione del diritto. Riflessioni su Interpretare e argomentare di Riccardo Guastini», Rivista di filosofia del diritto, 2 (2013/1), pp. 73-136.

VILLA, V., Conoscenza giuridica e concetto di diritto positivo, Giappichelli, Torino, 1993.

VILleY, M., «De l'indicatif dans le droit», Archives de Philosophie du droit, 19 (1974).

VIOLA, F., «Il futuro del diritto», Persona y Derecho, 79 (2018, II) [en este mismo número], pp. 9-36.

ZaCCARIa, G. (a cura di), Diritto positivo e positività del diritto, Giappichelli, Torino, 1991. 
0000 